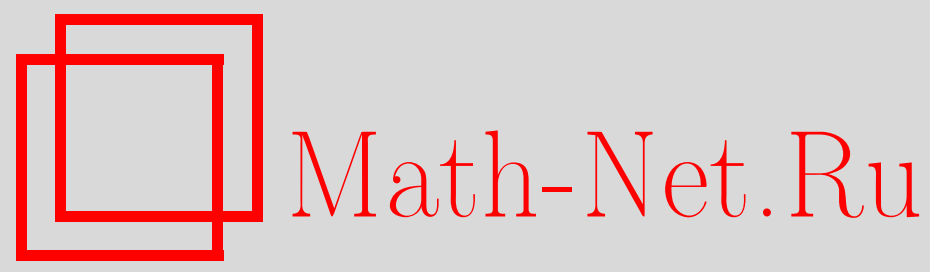

Д. А. Туганбаев, Мальцевские кольца, Дискрет. матем., 2008, том 20, выпуск 2, 63-81

DOI: https://doi.org/10.4213/dm1004

Использование Общероссийского математического портала Math-Net.Ru подразумевает, что вы прочитали и согласны с пользовательским соглашением http://www . mathnet.ru/rus/agreement

Параметры загрузки:

IP : 3.85 .73 .92

26 апреля 2023 г., 15:47:05 
УДК 512.55

\title{
Мальцевские кольца
}

\author{
() 2008 г. Д. А. Туганбаев
}

\begin{abstract}
В статье определяются мальцевские кольца. Класс всех мальцевских колец строго содержит кольца рядов Мальцева-Неймана, кольца косых формальных рядов Лорана и кольца формальных псевдодифференциальных операторов. Исследуются теоретикокольцевые свойства мальцевских колец. Оказывается, что кольца рядов МальцеваНеймана, кольца косых формальных рядов Лорана и кольца формальных псевдодифференциальных операторов имеют близкие теоретико-кольцевые свойства, связанные с существованием фильтрации по младшей степени ряда.
\end{abstract}

\section{1. Введение}

Начало использования колец косых рядов Лорана восходит к работам Шура, Диксона и Гильберта начала XX века. Например, при изучении независимости аксиом в геометрии Гильберт использовал кольцо косых рядов Лорана для построения тела, бесконечномерного над своим центром. Изучение колец рядов Лорана с произвольным кольцом коэффициентов было начато в конце 70-х начале 80-х годов Лоренцем [10], Рисманом [18] и Смитсом [20]. Техника использования колец рядов Лорана является удобным инструментом в теории колец. Например, в [11] Макар-Лиманов с помощью колец косых рядов Лорана от двух переменных показал, что кольцо частных алгебры Вейля содержит свободную некоммутативную подалгебру. В работе Гудерла и Смолла [6] кольца рядов Лорана используются для оценивания размерности Крулля и глобальной размерности нетеровых Р.І. колец.

Кольца рядов Лорана тесно связаны с кольцами рядов Мальцева-Неймана и кольцами обобщенных степенных рядов, интенсивно изучаемыми в последнее время. Напомним, что кольца рядов Мальцева-Неймана были определены А. И. Мальцевым в 1948 году в статье [12] для доказательства вложимости групповой алгебры над полем в тело (независимо в 1949 году эта конструкция была определена Б. Нейманом [14]). Среди многочисленных работ в этом направлении мы отметим работы Бергмана [2], Лоренца [10], Рисмана [18], Смитса [20] и Массона и Стаффорда [13]. Кольца обобщенных степенных рядов с показателями степени в упорядоченном моноиде в последние годы изучались многими авторами (см., например, книгу [1] и статьи [3, 17, 8, 9]). Теоретико-кольцевые свойства колец, близких к кольцам рядов Лорана, систематически исследуются в работах [21]-[33].

Алгебра псевдодифференциальных операторов $A\left(\left(\delta^{-1}\right)\right)$ была введена Шуром в 1905 году в работе [19] и с тех пор неоднократно использовалась в различных разделах математики. Поскольку в работе рассматриваются лишь теоретико-кольцевые свойства колец псевдодифференциальных операторов, мы не излагаем здесь историю применения псевдодифференциальных операторов в математике и не приводим соответствующие работы, 
не относящиеся к структурной теории колец. Отметим только работу А. Н. Паршина [15], в которой автор развил алгебраическую теорию колец формальных псевдодифференциальных операторов от нескольких переменных. В этой же работе используются итерированные кольца косых рядов Лорана. В структурной теории колец кольца псевдодифференциальных операторов используются для конструктивизации вычислений в алгебрах дифференциальных операторов (см. [5]), а также как источник многочисленных примеров (см., например, [7]). Если кольцо псевдодифференциальных операторов имеет правую размерность Крулля, то оно является нетеровым справа кольцом [24].

Все кольца предполагаются ассоциативными и с ненулевой единицей, но не обязательно коммутативными. В статье используются базовые сведения из теории колец, которые можно найти, например, в [4].

\section{2. Фильтрованные кольца}

Пусть $G$ - произвольная группа. Единицу $1_{G}$ группы $G$ будем обозначать просто 1 . Тогда кольцо $R$ называется $G$-фильтрованным кольцом, если в нем задан набор подгрупп по сложению $\left\{U_{g}\right\}$ (где $g-$ произвольный элемент группы $G$ ) такой, что для любых $g$ и $h$ из $G$ выполнено включение $U_{g} U_{h} \subseteq U_{g h}$ и для единицы $1_{R}$ кольца $R$ выполнено включение $1_{R} \in U_{1}$. В этом случае будем также говорить, что на кольце $R$ задана фильтрация $\left\{U_{g}\right\}$. Фильтрация называется исчерпывающей, если объединение $U_{g}$ по всем $g$ из $G$ составляет все кольцо $R$. Фильтрация называется отделимой, если пересечение $U_{g}$ по всем $g$ из $G$ состоит из одного нуля.

Отметим, что $U_{1}$ всегда является унитарным подкольцом кольца $R$.

Группа $G$ называется упорядоченной, если она удовлетворяет следующим условиям:

(1) множество $G$ линейно упорядочено, то есть все элементы $G$ сравнимы между собой относительно некоторого рефлексивного, антисимметричного и транзитивного отношения $\leqslant$;

(2) для любых элементов $x, y, z \in G$ из $x \leqslant y$ следует $x z \leqslant y z$ и $z x \leqslant z y$.

Если на кольце $R$ задана фильтрация $\left\{U_{g}\right\}$ упорядоченной группой $G$ и для каждой пары элементов $g$ и $h$ из $G$ таких, что $g \leqslant h$, справедливо включение $U_{h} \subseteq U_{g}$, то будем говорить, что на $R$ задана упорядоченная фильтрация. Очевидно, что для любого $g \geqslant 1$ множество $U_{g}$ является двусторонним идеалом в кольце $U_{1}$.

Для упорядоченной фильтрации будем обозначать через $U_{g}+$ объединение $U_{h}$ по всем $h>g$. Очевидно, $U_{g}+\subseteq U_{g}$. Аналогично, $U_{g}$ - будет обозначать пересечение всех $U_{h}$ по всем $h<g$. Очевидно, $U_{g} \subseteq U_{g-}$. Будем говорить, что упорядоченная фильтрация является строго исчерпывающей сверху, если объединение $U_{g} \backslash U_{g}+$ по всем $g$ из $G$ составляет все кольцо $R$ за исключением нулевого элемента. Легко видеть, что всякая строго исчерпывающая сверху фильтрация является исчерпывающей и отделимой. В случае, когда $G-$ свободная циклическая группа, верно и обратное утверждение, что всякая отделимая исчерпывающая фильтрация является строго исчерпывающей сверху.

В случае строго исчерпывающей сверху фильтрации для всякого $r$ из $R$ будем обозначать $\operatorname{deg} r$ такой элемент $g \in G$, что $r$ лежит в $U_{g} \backslash U_{g+}$. Из определения строго исчерпывающей сверху фильтрации видно, что для всякого ненулевого элемента $r$ из $R$ элемент $\operatorname{deg} r$ из $G$ определен, причем единственным образом. Будем считать, что $\operatorname{deg} 0=+\infty$. Элемент $\operatorname{deg} r \in G$ будем также называть младшей степенью элемента $r$. Легко видеть, что для любых $r_{1}, r_{2}$ из $R$ выполнено неравенство $\operatorname{deg} r_{1} r_{2} \geqslant \operatorname{deg} r_{1} \operatorname{deg} r_{2}$. 
Из того, что единица $1_{R}$ кольца $R$ лежит в $U_{1}$, и из неравенства $\operatorname{deg} 1_{R} \geqslant \operatorname{deg} 1_{R} \operatorname{deg} 1_{R}$ вытекает, что $\operatorname{deg} 1_{R}=1$.

Будем называть обратимый элемент $y \in R$ сильно обратимым, если для него выполнено равенство $\operatorname{deg} y^{-1}=(\operatorname{deg} y)^{-1}$. Нетрудно видеть, что для любого сильно обратимого элемента $y \in R$ и любого элемента $r \in R$ выполнены равенства $\operatorname{deg} y r=\operatorname{deg} y \operatorname{deg} r$ и $\operatorname{deg} r y=\operatorname{deg} r \operatorname{deg} y$. Непосредственно проверяется, что все сильно обратимые элементы фильтрованного кольца образуют группу.

\section{3. Сильно фильтрованные кольца}

Определение 1. Пусть $G$ - упорядоченная группа. Тогда кольцо $R$ называется мальцевским кольцом, если на нем задана фильтрация $\left\{U_{g}\right\}$ так, что выполнены следующие свойства:

(i) $\left\{U_{g}\right\}-$ строго исчерпывающая сверху фильтрация;

(ii) для любого $g$ из $G$ найдется хотя бы один сильно обратимый элемент $y \in U_{g}$;

(iii) для любого множества $\left\{r_{\alpha} \mid \alpha \in \Omega\right\}$ элементов кольца $R$ найдется хотя бы один элемент $r \in R$ (обобщенная сумма элементов $r_{\alpha}$ ) такой, что если для некоторого $g \in G$ все элементы $\left\{r_{\alpha}\right\}$ (кроме конечного числа $r_{1}, \ldots, r_{n}$ ) лежат в $U_{g}$, то и разность $r-\sum_{i=1}^{n} r_{n}$ (разность между полной и частичной суммой) лежит в $U_{g}$;

(iv) канонический кольцевой гомоморфизм кольца $U_{1}$ на свое факторкольцо $A=U_{1} / U_{1+}$ расщепляется, то есть существует вложение $\pi: A \rightarrow U_{1}$ такое, что его композиция с каноническим гомоморфизмом $U_{1}$ на $A$ дает тождественный автоморфизм кольца $A$. Кроме того, для любого $a$ из $A$ выполнены равенства $\pi(a) R \cap U_{1}=\pi(a) U_{1}$ и $R \pi(a) \cap U_{1}=U_{1} \pi(a)$.

Кольцо, в котором выполнены условия (i)-(ii), но не обязательно выполнены условия (iii)-(iv), будем называть сильно фильтрованным кольцом или кольцом, на котором задана сильная фильтрация. Кольцо, в котором выполнены условия (i)-(iii), но не обязательно выполнено условие (iv), будем называть обобщенным мальцевским кольцом.

В рамках данной работы главным изучаемым свойством, позволяющим получить наиболее нетривиальные результаты, является условие (iii). Наличие бесконечного суммирования отличает кольца рядов от колец многочленов. В общем случае, обобщенная сумма из условия (iii) определена не единственным образом.

Ниже будет показано, что примерами мальцевских колец являются кольца рядов Лорана, кольца косых рядов Лорана (где фильтрация группой $\mathbf{Z}$ вводится по младшей степени формального ряда), кольца псевдодифференциальных операторов (где фильтрация группой $\mathbf{Z}$ вводится по старшей степени формального ряда) и кольца рядов МальцеваНеймана. Понятие мальцевского кольца позволяет доказать ряд общих утверждений, которые будут справедливы для всех перечисленных классов колец.

Непосредственно проверяется, что для всякого кольца $R$ с упорядоченной фильтрацией $U_{1}-$ унитарное подкольцо в $R$, а $U_{1+}-$ двусторонний идеал в $U_{1}$. Поэтому можно рассмотреть факторкольцо $A=U_{1} / U_{1+}$, которое будет называться кольцом коэффициентов кольца с упорядоченной фильтрацией. Ниже будет доказано, что кольцо косых рядов Лорана, кольцо псевдодифференциальных операторов и кольцо рядов Мальцева-Неймана являются мальцевскими кольцами, так что понятие кольца коэффициентов в них совпадает с обычным определением.

3 Дискретная математика, т.20 №2 
Нам потребуются следующие обозначения.

Пусть $R$ - кольцо с упорядоченной фильтрацией, а $\left\{U_{g}\right\}-$ набор множеств в нем, как в определении. Тогда для каждого элемента из $U_{1}$ назовем его свободным членом его образ при каноническом гомоморфизме $U_{1}$ на $A=U_{1} / U_{1+}$. В случае кольца рядов Лорана это определение совпадает с естественным определением свободного члена (если рассматривать фильтрацию группой $\mathbf{Z}$ по младшей степени ряда). Свободный член определен только для элементов из $U_{1}$. Свободный член элемента $f$ будет обозначаться через $\hat{f}$. Легко видеть, что свободный член суммы (произведения) двух элементов из $U_{1}$ равен сумме (произведению) их свободных членов.

В сильно фильтрованном кольце элементы с младшей степенью 1 - это в точности все элементы из $U_{1}$ с ненулевым свободным членом. Из определения сильно фильтрованного кольца вытекает, что каждый ненулевой элемент $f$ кольца $R$ может быть представлен в виде произведения вида $u y$, где $u \in U_{1}$ - элемент с ненулевым свободным членом, $y-$ сильно обратимый элемент.

Для каждого подмножества $P$ кольца $R$ с упорядоченной фильтрацией обозначим через $\lambda(P)$ образ множества $U_{1} \cap P$ при каноническом гомоморфизме $U_{1}$ на $U_{1} / U_{1+}$ (то есть $\lambda(P)$ - это множество всех свободных членов всех элементов из $\left.U_{1} \cap P\right)$. Непосредственно проверяется, что если $P$ - левый (правый) идеал кольца $R$, то $\lambda(P)$ является левым (правым) идеалом кольца коэффициентов $A=U_{1} / U_{1+}$. Таким образом, отображение $\lambda$ осуществляет отображение решетки левых (правых) идеалов кольца $R$ в решетку левых (правых) идеалов кольца $A$, причем это отображение сохраняет отношение включения. В сильно фильтрованном кольце $R$, кроме того, если $P$ - ненулевой правый (левый) идеал, то $\lambda(P)-$ ненулевой правый (левый) идеал кольца коэффициентов (действительно, если $f \in P$ и $g=\operatorname{deg} f$, а $y-$ сильно обратимый элемент из $U_{g}$, то $f y^{-1} \in U_{1} \cap P$ или $y^{-1} f \in U_{1} \cap P$, при этом элементы $f y^{-1}$ и $y^{-1} f$ имеют ненулевые свободные члены).

Если $y$ - сильно обратимый элемент сильно фильтрованного кольца $R$, то внутренний автоморфизм $r \rightarrow y r y^{-1}$ сохраняет младшую степень элементов и, в частности, сохраняет на месте $U_{1}$ и $U_{1+}$. Поэтому он индуцирует автоморфизм $\varphi_{y}$ кольца коэффициентов $U_{1} / U_{1+}$. Будем говорить, что он индуцирует скручивающий автоморфизм $\varphi_{y}$ кольца коэффициентов $A$.

\section{4. Свойства сильно фильтрованных колец}

Докажем теперь некоторые вспомогательные утверждения о сильно фильтрованных кольцах.

Лемма 1. Пусть $R$ - сильно фильтрованное кольц̧о, $A-$ его кольц̧о коэффициентов и $A$ - область. Тогда для любых двух ненулевых элементов $r_{1}$ u $r_{2}$ из $R$ младшая степень $u x$ произведения равна произведению их младших степеней.

Доказательство. Действительно, если $g$ - младшая степень элемента $r_{1}$, а $h$ - младшая степень элемента $r_{2}$, то найдутся такие элементы $s_{1}$ и $s_{2}$ из $U_{1} \backslash U_{1+}$, что $r_{1}=y_{g} s_{1}$ и $r_{2}=s_{2} y_{h}$, где $y_{g}$ и $y_{h}-$ сильно обратимые элементы, как в определении сильно фильтрованного кольца. Поскольку элементы $s_{1}$ и $s_{2}$ имеют ненулевые свободные члены, их произведение $s_{1} s_{2}$ имеет ненулевой свободный член и, следовательно, лежит в $U_{1} \backslash U_{1+}$.

Произведение $r_{1} r_{2}=y_{g} s_{1} s_{2} y_{h}$ лежит в $U_{g h}$. Допустим, оно лежит также в $U_{g h+}$, тогда произведение $s_{1} s_{2}=y_{g}^{-1} r_{1} r_{2} y_{h}^{-1}$ лежит в $U_{1+}$, чего не может быть. Таким образом, произведение $r_{1} r_{2}=y_{g} s_{1} s_{2} y_{h}$ лежит в $U_{g h} \backslash U_{g h+}$, что и требовалось доказать. 
Для всякого сильно фильтрованного кольца $R$ с группой $G$ отображение $r \rightarrow \operatorname{deg} r$, ограниченное на множество сильно обратимых элементов $R^{*}$, является гомоморфизмом группы $R^{*}$ на группу $G$, причем образ гомоморфизма совпадает со всей группой $G$. Поэтому группа $G$ изоморфна факторгруппе группы $R^{*}$ по подгруппе $R_{1}^{*}$ всех сильно обратимых элементов кольца $R$ с младшей степенью $1_{G}$. Поэтому структуру мальцевского кольца на каком-то кольце $R$ можно задавать не непосредственно через группу $G$, а путем указания группы сильно обратимых элементов $R^{*}$ и множества $U_{1}$ (указывая таким образом и подгруппу $R_{1}^{*}$ ). При этом достаточно указать не всю группу сильно обратимых элементов $R^{*}$, а некоторую ее репрезентативную подгруппу $R^{\prime}$. Приведем точное определение и докажем соответствующее утверждение.

Для всякого сильно фильтрованного кольца $R$ с группой $G$ будем называть подмножество $R^{\prime} \subset R$ репрезентативной группой, если $R^{\prime}$ является подгруппой группы сильно обратимых элементов кольца $R$ и для каждого элемента $g$ из $G$ в множестве $R^{/}$найдется элемент степени $g$.

Замечание 1. В кольце рядов Лорана (с фильтрацией по младшим степеням рядов) или кольце многочленов Лорана от одной переменной $x$ репрезентативной группой всегда является множество одночленов, являющихся степенями переменной $x$ с единичным коэффициентом.

Лемма 2. Пусть $R$ - кольи̧о, $R^{\prime}-$ подгруппа $U(R)$ (группь обратимых элементов кольц̧а $R)$. Пусть $U_{1}$ - унитарное подкольичо в $R$ такое, что для каждого элемента $r$ из $R^{\prime}$ справедливо включение $r \in U_{1}$ или $r^{-1} \in U_{1}$, и при этом $r U_{1} r^{-1} \subseteq U_{1}$. Пусть для каждого ненулевого $r \in R$ среди всех множеств вида $r^{\prime} U_{1}$ (где $r^{\prime}-$ произвольный элемент из $\left.R^{\prime}\right)$, содержащих $r$, найдется наименьшее в смысле упорядочения по включению. Пусть, наконец, $U_{1} R^{\prime}=R$ или $R^{\prime} U_{1}=R$. Тогда сущчествуют группа $G$ и сильная фильтрация $\left\{U_{g}\right\}$ такие, что $U_{1_{G}}=U_{1}$ и множество $R^{/}$является репрезентативной подгруппой кольца $R$.

Доказательство. Из условия $r U_{1} r^{-1} \subseteq U_{1}$ сразу вытекает, что требуемые в условии равенства $R^{\prime} U_{1}=R$ и $U_{1} R^{\prime}=R$ равносильны.

Пусть $R_{1}^{\prime}$ - множество таких элементов $r \in R^{\prime}$, что $r \in U_{1}$ и $r^{-1} \in U_{1}$. Легко видеть, что $R_{1}^{\prime}$ - подгруппа в $R^{\prime}$. Докажем, что она нормальна. Действительно, пусть $r_{1}-$ произвольный элемент из $R_{1}^{\prime}$, а $r$ - произвольный элемент из $R$. Тогда $r r_{1} r^{-1} \in r U_{1} r^{-1} \subseteq U_{1}$ и $r r_{1}^{-1} r^{-1} \in r U_{1} r^{-1} \subseteq U_{1}$, то есть и элемент $r r_{1} r^{-1}$ и его обратный лежат в $U_{1}$. Это и означает, что элемент $r r_{1} r^{-1}$ лежит в $R_{1}^{\prime}$.

Тогда можно рассмотреть факторгруппу $G \equiv R^{/} / R_{1}^{\prime}$. Для каждого $g=r R_{1}^{\prime}$ из группы $G$ положим $U_{g} \equiv r U_{1}$. Это определение корректно (не зависит от выбора конкретного $r$ ), так как $r U_{1} \subseteq r R_{1}^{\prime} U_{1} \subseteq r U_{1} U_{1}=r U_{1}$. Пусть $g=r R_{1}^{\prime}$ и $h=s R_{1}^{\prime}-$ два произвольных элемента группы $G$. Тогда $U_{g} U_{h}=r U_{1} s U_{1}=r s\left(s^{-1} U_{1} s\right) U_{1} \subseteq r s U_{1} U_{1}=r s U_{1}=U_{g h}$, таким образом, множества $U_{g}$ действительно задают фильтрацию на кольце $R$.

Для любой пары элементов $r$ и $s$ из $R^{/}$по условию справедливо включение $s^{-1} r \in U_{1}$ или включение $r^{-1} s \in U_{1}$. В первом случае получаем, что $r \in s U_{1}$, во втором $s \in r U_{1}$. Поэтому для любых $g$ и $h$ из $G$ либо $U_{g}$ лежит в $U_{h}$, либо $U_{h}$ лежит в $U_{g}$. Поэтому набор множеств $U_{g}$ линейно упорядочен по включению. Непосредственно проверяется, что если $U_{g}=U_{h}$, то $g=h$ (действительно, если $r \in s U_{1}$ и $s \in r U_{1}$, то и $s^{-1} r \in U_{1}$ и $r^{-1} s \in U_{1}$, откуда $r^{-1} s \in R_{1}^{\prime}$ ). Это позволяет, воспользовавшись линейной упорядоченностью набора $U_{g}$, задать соответствующий линейный порядок на группе $G$ так, чтобы фильтрация стала упорядоченной. Действительно, достаточно положить, что $g \leqslant h$ тогда и только тогда, 
когда $U_{h} \subseteq U_{g}$. Из того, что $R^{/} U_{1}=R$, следует, что построенная фильтрация является исчерпывающей.

Докажем, что построенная фильтрация является строго исчерпывающей сверху. Действительно, пусть $r$ - произвольный ненулевой элемент кольца $R$. Тогда по условию среди всех множеств $U_{g}$ существует наименьшее (в смысле упорядоченности по включению), содержащее $r$. Непосредственно проверяется, что элемент $g$ группы $G$ и будет младшей степенью элемента $r$ (то есть $r \in U_{g} \backslash U_{g+}$ ).

Непосредственно проверяется, что для каждого элемента $r^{\prime}$ из $R^{\prime}$ его младшей степенью является его образ при каноническом гомоморфизме $R^{\prime}$ на $G$. Отсюда вытекает, что все элементы $R^{\prime}$ являются сильно обратимыми. Поэтому построенная фильтрация является также и сильной (то есть в каждом множестве $U_{g}=r^{\prime} U_{1}$ найдется хотя бы один сильно обратимый элемент, а именно $r^{\prime}$ ). Наконец, по построению $U_{1_{G}}=U_{1}$. Из того, что множества $U_{g}$ выбирались равными $r^{\prime} U_{1}$, вытекает, что множество всех элементов $r^{\prime}$ (то есть $R^{\prime}$ ) будет репрезентативной группой.

Непосредственно проверяется еще одно вспомогательное утверждение о репрезентативной группе.

Лемма 3. Пусть $R$ - сильно фильтрованное кольияо с фильтрацией $\left\{U_{g}\right\}$, где $g$ - элементы упорядоченной группь $G$. Пусть $R^{\prime}$ - репрезентативная группа в нем. Тогда множество $R_{1}^{\prime}$, состоящее из всех элементов $R^{\prime}$ с единичной младшей степенью, является нормальной подгруппой в группе $R^{\prime}$, причем факторгруппа $R^{\prime} / R_{1}^{\prime}$ изоморфна $G$, где в качестве изоморфизма можно взять отображение, которое элементу $r^{\prime} R_{1}^{\prime}$ сопоставляет младшую степень $\operatorname{deg} r^{\prime}$ элемента $r^{\prime}$. Кроме того, для элементов $R^{\prime}$ справедливо утверждение, что $r_{1}^{\prime}$ тогда и только тогда лежит в $r_{2}^{\prime} U_{1}$, когда для их младиих степеней $g_{1}$ и $g_{2}$ выполнено неравенство $g_{1} \geqslant g_{2}$.

Докажем теперь утверждение, которое покажет, что конструкция сильно фильтрованного кольца допускает последовательное итерирование с сохранением своих свойств (так, кольцо многочленов Лорана над кольцом многочленов Лорана также является кольцом многочленов Лорана от двух переменных над исходным кольцом).

Предложение 1. Пусть $S-$ сильно фильтрованное кольцุо с кольцом коэффициентов $R$ и фильтрацией $\left\{U_{g}\right\}$, где $g$ - элементы упорядоченной группь $G$. Пусть $R-$ сильно фильтрованное кольцо с кольцом коэффициентов $A$ и фильтрацией $\left\{V_{h}\right\}$, где $h-$ элементы упорядоченной группь $H$. Пусть в $S$ существует репрезентативная группа $S^{\prime}$ (в смысле фильтрации $\left\{U_{g}\right\}$ ). Пусть, к тому же, множество $R^{\prime}$, образ $S_{1}^{\prime}$ (множества всех элементов из $S^{\prime}$ с младшей степенью $1_{G}$ ) при каноническом гомоморфизме на кольцо коэффициентов $R$, является репрезентативной группой в смысле фильтрации $\left\{V_{h}\right\}$. Пусть при этом для каждого $s \in S^{\prime}$ скручивающчий автоморфизм $\varphi_{s}$ кольияа $R$, индуцированный элементом $s$, сохраняет младшую степень всех элементов $R$ (в смысле фильтращии $\left.\left\{V_{h}\right\}\right)$. Тогда существует упорядоченная группа $G \circ H$ такая, что в ней есть нормальная подгруппа, изоморфная $H$, факторгруппа по которой изоморфна $G$, а отношение порядка в $G$ ○ этом $S$ является сильно фильтрованныл кольцом с фильтрацией $\{W\}$ группой $G \circ H$, а кольио коэффициентов изоморфно А. Все элементы из $S^{\prime}$ при этом являются сильно обратимыми в смысле новой фильтрации $\{W\}$.

Доказательство. Определим вначале $W_{1}$ как множество всех таких элементов из $U_{1}$, свободные члены (в смысле фильтрации $\left.\left\{U_{g}\right\}\right)$ которых лежат в $V_{1}$. Проверим все условия леммы 2. 
Проверим, что $S=S^{\prime} W_{1}$. Действительно, из того, что $R^{\prime}$, образ $S_{1}^{\prime}$, является репрезентативной группой в кольце $R$, вытекает равенство $S_{1}^{/} W_{1}+U_{1+}=U_{1}$. Поскольку множество $U_{1}$ лежит в множестве $W_{1}$, отсюда вытекает равенство $S_{1}^{/} W_{1}=U_{1}$. Но тогда $\left.S=S^{/} U_{1}=S^{/} S_{1}^{/} U_{1}\right) W_{1}=S^{/} W_{1}$.

Из условия, что для всех элементов $s^{\prime}$ из $S^{/}$скручивающий автоморфизм $\varphi_{s^{\prime}}$ сохраняет младшую степень всех элементов $R$, также вытекает, что $s^{\prime} W_{1} s^{\prime-1} \subseteq W_{1}$. Все элементы $s^{\prime}$ из $S^{\prime}$ имеют младшую степень (в смысле фильтрации $\left.\left\{U_{g}\right\}\right)$ либо отличную от единицы (и тогда либо $s^{\prime}$, либо $s^{\prime-1}$ лежит в $U_{1}+W_{1}$ ), либо равную единице (и тогда младший член $s^{\prime}$ или младший член $s^{\prime-1}$ лежит в $V_{1}$, из чего также вытекает, что или $s^{\prime}$ или $s^{\prime-1}$ лежит в $\left.W_{1}\right)$.

Остается проверить, что для каждого ненулевого элемента $s$ из кольца $S$ существует наименьшее (в смысле упорядоченности по включению) множество $s^{\prime} W_{1}$, где элемент $s^{\prime}$ лежит в $S^{\prime}$. Действительно, поскольку $S^{\prime}-$ репрезентативная группа в $S$, элемент $s$ можно представить в форме $s_{1}^{\prime} u_{1}$, где $u_{1}$ лежит в $U_{1}$ и имеет ненулевой свободный член $\hat{u}_{1}$. В свою очередь этот свободный член имеет аналогичное представление в кольце $R$, откуда получаем, что $s \in s_{1}^{\prime}\left(s_{2}^{\prime} w_{1}+U_{1+}\right)$, где $w_{1}$ лежит в $W_{1}$, причем свободный член (в смысле кольца $S$ ) элемента $w_{1}$ имеет ненулевой свободный член (в смысле кольца $R$ ). Отсюда и вытекает необходимое утверждение, если положить $s^{\prime}=s_{1}^{\prime} s_{2}^{\prime}$.

Тогда можно применить лемму 2 и получить в кольце $S$ сильную фильтрацию $\left\{W_{f}\right\}$ и группу $F$ такие, что множество $S^{/}$будет репрезентативной группой. Остается проверить искомые свойства группы $G \circ H \equiv F$.

Действительно, по лемме 3 группа $F$ изоморфна факторгруппе $S^{\prime}$ по подгруппе $S_{1}^{/} 1$, состоящей из всех элементов $S^{\prime}$, имеющих единичную младшую степень (в смысле новой фильтрации $\left.\left\{W_{f}\right\}\right)$. По той же лемме группа $G$ изоморфна факторгруппе $S^{\prime}$ по подгруппе $S_{1}^{\prime}$, а группа $H$ изоморфна факторгруппе $R^{\prime}$ по подгруппе $R_{1}^{\prime}$. При этом выполнена цепочка вложений $S_{1}^{\prime} 1 \subseteq S_{1}^{\prime} \subseteq S^{\prime}$. Остается отметить, что $R^{\prime}$ и $R_{1}^{\prime}$ являются образами $S_{1}^{\prime}$ и $S_{1}^{/} 1$ соответственно при каноническом гомоморфизме $U_{1}$ на $R$. С учетом того, что прообраз единицы при этом гомоморфизме лежит в $S_{1}^{/}$, отсюда и получается искомое утверждение о том, что $G \cong S^{/} / S_{1}^{\prime}$ и $H \cong S_{1}^{\prime} / S_{1}^{\prime} 1$. Необходимые свойства порядка, заданного на этой группе, проверяются непосредственно с учетом леммы 3.

\section{5. Обобщенные мальцевские кольца}

Для обобщенных мальцевских колец справедливо следующее важное утверждение.

Лемма 4. Пусть $R$ - обобщенное мальцевское кольияо, $A-$ его кольцуо коэффициентов, $P$ - правый идеал в $R$. Допустим, что существуют такие элементы $f_{1}, f_{2}, \ldots, f_{n}$ из $P \cap U_{1}$, что свободные члень всех элементов из $P \cap U_{1}$ лежат в правом идеале, порожденном свободными членами элементов $f_{1}, f_{2}, \ldots, f_{n}$ (то есть для каждого элемента $g$ из $P \cap U_{1}$ справедливо включение $\hat{g} \in \hat{f}_{1} A+\hat{f}_{2} A+\ldots+\hat{f}_{n} A$, где $\hat{f}_{i}-$ свободный член элемента $f_{i}$, a $\hat{g}$ - свободный член элемента $g$ ). Тогда правый идеал $P$ порождается $n$ элементами $f_{1}, f_{2}, \ldots, f_{n}$.

Доказательство. Выберем для каждого $g \in G$ какой-либо сильно обратимый элемент $y_{g}$ из $U_{g}$, существующий по условию (ii).

Обозначим через $Q$ правый идеал $f_{1} R+f_{2} R+\ldots+f_{n} R$ кольца $R$. Так как все элементы $f_{i}$ лежат в идеале $P$ и $Q=f_{1} R+f_{2} R+\ldots+f_{n} R$, справедливо включение 
$Q \subseteq P$. Допустим, что утверждение леммы не верно. Тогда существует элемент $h \in P$ такой, что $h \notin Q$. Без ограничения общности можно считать, что $h \in U_{1}$ (если это не так, то можно домножить $h$ на сильно обратимый элемент $y_{\operatorname{deg} h^{-1}}$ ).

На множестве всех возможных наборов $\left\{r_{1}, r_{2}, \ldots, r_{n}\right\}$ введем отношение частичного порядка. Скажем, что набор $\left\{s_{1}, s_{2}, \ldots, s_{n}\right\}$ больше набора $\left\{r_{1}, r_{2}, \ldots, r_{n}\right\}$ тогда и только тогда, когда младшая степень $g_{s}$ элемента $h-f_{1} s_{1}-f_{2} s_{2}-\ldots-f_{n} s_{n}$ больше, чем младшая степень $g_{r}$ элемента $h-f_{1} r_{1}-f_{2} r_{2}-\ldots-f_{n} r_{n}$ и при этом для всех $k$ справедливо включение $s_{k}-r_{k} \in U_{g_{r}}$.

Применим лемму Цорна. Для этого надо доказать, что любая возрастающая цепь имеет верхнюю грань. Действительно, пусть $\left\{r_{1}^{(i)}, r(i)_{2}, \ldots, r(i)_{n}\right\}$ при $0<i<\infty-$ возрастающая цепь таких наборов и $h^{(i)}=h-f_{1} r_{1}^{(i)}-f_{2} r_{2}^{(i)}-\ldots-f_{n} r_{n}^{(i)}$. Тогда для каждого натурального $i$ и каждого $k, 1 \leqslant k \leqslant n$, по условию справедливо включение $r_{k}^{(i+1)}-r_{k}^{(i)} \in U_{\operatorname{deg} h^{(i)}}$. Для каждого $k$ существует обобщенная бесконечная сумма элементов $r_{k}^{(i+1)}-r_{k}^{(i)}$ по всем $1 \leqslant i<\infty$. Поскольку обобщенная бесконечная сумма может быть определена не единственным образом, выберем произвольную. Прибавим к этой сумме $r_{k}^{(1)}$ и обозначим полученный результат $r_{k}$. Докажем тогда, что набор $\left\{r_{1}, r_{2}, \ldots, r_{n}\right\}$ и является искомой верхней гранью. Действительно, для всякого $k$ и всякого $i$ элемент $r_{k}-r_{k}^{(i)}$ по определению обобщенной бесконечной суммы имеет младшую степень не ниже, чем у элемента $r_{k}^{(i+1)}-r_{k}^{(i)}$, а это и означает, что элемент $r_{k}-r_{k}^{(i)}$ лежит в $U_{\operatorname{deg} h^{(i)}}$. Остается доказать, что для каждого $i$ младшая степень $g_{r}$ элемента $h-f_{1} r_{1}-f_{2} r_{2}-\ldots-f_{n} r_{n}$ больше, чем младшая степень $g_{r}(i)$ элемента $h-f_{1} r_{1}^{(i)}-f_{2} r_{2}^{(i)}-\ldots-f_{n} r_{n}^{(i)}$. Достаточно доказать нестрогое неравенство, поскольку последовательность $g_{r}(i)$ строго возрастает, и если элемент $g_{r}$ нестрого больше каждого ее члена, то он и строго больше каждого из них. Для этого достаточно доказать, что младшая степень элемента $s=\left(h-f_{1} r_{1}-f_{2} r_{2}-\ldots-f_{n} r_{n}\right)-\left(h-f_{1} r_{1}^{(i)}-f_{2} r_{2}^{(i)}-\ldots-f_{n} r_{n}^{(i)}\right)$ не ниже $g_{r^{(i)}}$. Но $s=f_{1}\left(r_{1}^{(i)}-r_{1}\right)+\ldots+f_{n}\left(r_{n}^{(i)}-r_{n}\right)$, так что искомое утверждение следует из включений $f_{i} \in U_{1}$ и $r_{k}-r_{k}^{(i)} \in U_{g_{r}(i)}$.

Из леммы Цорна получаем, что существует максимальный набор $\left\{r_{1}, r_{2}, \ldots, r_{n}\right\}$. Так как $h$ не лежит в $Q=f_{1} R+f_{2} R+\ldots+f_{n} R$, элемент $s=h-f_{1} r_{1}-f_{2} r_{2}-\ldots-f_{n} r_{n}$ отличен от нуля. Найдем сильно обратимый элемент $y$ из $U_{\operatorname{deg} s}$. Тогда $s y^{-1}$ лежит в $U_{1} \subseteq P$. Тогда свободный член $s_{0}$ элемента $s y^{-1}$ лежит в $\lambda P$, и поэтому элемент $s_{0}$ кольца коэффициентов может быть представлен в виде $\hat{f}_{1} a_{1}+\hat{f}_{2} a_{2}+\ldots+\hat{f}_{n} a_{n}$. Пусть каждый элемент $a_{i} \in A$ является свободным членом какого-то элемента $r_{i}^{\prime} \in U_{1}$. Тогда свободный член элемента $s y^{-1}-f_{1} r_{1}^{\prime}-\ldots-f_{n} r_{n}^{\prime}$ равен $s_{0}-s_{0}=0$. Поэтому младшая степень элемента $s-f_{1} r_{1}^{\prime} y-\ldots-f_{n} r_{n}^{\prime} y=h-f_{1}\left(r_{1}+r_{1}^{\prime} y\right)-\ldots-f_{n}\left(r_{n}+r_{n}^{\prime} y\right)$ строго больше младшей степени $y$, которая равна младшей степени $s$. С учетом того, что $r_{i}^{\prime} y$ лежит в $U_{\operatorname{deg} s}$ для всех $i$, получаем противоречие с тем, что $\left\{r_{1}, r_{2}, \ldots, r_{n}\right\}$ - наибольший набор. Полученное противоречие и завершает доказательство.

Замечание 2. Аналог леммы 4 для левых идеалов также верен.

В качестве простого следствия этой леммы можно получить следующее утверждение (его частные случаи для колец косых рядов Лорана и колец псевдодифференциальных операторов хорошо известны).

Предложение 2. Пусть $R$ - обобщуенное мальцевское кольцьо, a $A$ - его кольцио коэффициентов. Тогда если свободный член элемента $r \in U_{1}$ обратим справа (слева) в кольце 
$A$, то и сам элемент $r$ обратим справа (слева) в кольце $R$. Кроме того, если $A-$ тело, то и $R-$ тело.

Доказательство. Действительно, пусть свободный член элемента $r$ обратим справа. Тогда применим лемму 4, где положим $n=1, f_{1}=r, P=R$, и получим, что $R=P=r R$, то есть, что элемент $r$ обратим справа.

Пусть теперь $A$ - тело, тогда из уже доказанного вытекает, что всякий элемент $u$ из $U_{1}$ с ненулевым свободным членом обратим справа (и слева). Но всякий ненулевой элемент $r$ кольца $R$ может быть представлен в виде произведения $u y$, где $u \in U_{1}$ имеет ненулевой свободный член, а $y-$ сильно обратимый элемент. Поэтому $r$ является произведением двух обратимых элементов и, следовательно, обратим.

В связи с предложением 2 заметим, что мальцевское кольцо является телом тогда и только тогда, когда его кольцо коэффициентов является телом (см. предложение 5). Для обобщенных мальцевских колец это неверно, соответствующий пример будет построен ниже (кольцо дробных $p^{n}$-адических чисел $Q_{p^{n}}$, при $n>1$, которое является телом в то время как его кольцо коэффициентов не является областью).

Для облегчения построения примеров мальцевских и обобщенных мальцевских колец покажем, что в условии (iii) определения мальцевского кольца достаточно проверять существование обобщенной суммы только для счетных множеств элементов специального вида.

Лемма 5. Пусть $R-$ сильно фильтрованное кольцо с фильтрацией $\left\{U_{g}\right\}$. Пусть для любой бесконечной последовательности ненулевых элементов $r_{1}, r_{2}, \ldots$ такой, что младиие степени $\operatorname{deg} r_{1}, \operatorname{deg} r_{2}, \ldots$ образуют строго возрастаюшую последовательность, существует элемент $r$ такой, что для всех натуральных $n$ выполнено неравенство

$$
\operatorname{deg} r-\sum_{i=1}^{n} \operatorname{deg} r_{i} \geqslant \operatorname{deg} r_{n+1} .
$$

Тогда элемент $r$ является обобщенной суммой элементов $r_{1}, r_{2}, \ldots$, и обобщенная сумма существует для любых множеств элементов из $R$, то есть $R$ является обобщенным мальцчевским кольцчом.

Доказательство. Тот факт, что элемент $r$ является обобщенной суммой элементов $r_{1}, r_{2}, \ldots$, проверяется непосредственно.

Отметим некоторые общие свойства обобщенной суммы. В любом сильно фильтрованном кольце, если существует элемент $a$, обобщенная сумма набора элементов $A$, и элемент $b$, обобщенная сумма набора элементов $B$, то существует по крайней мере одна обобщенная сумма объединенного набора $A \cup B$, равная $a+b$. Поэтому, если удается разбить множество элементов на две части, для каждой из которых обобщенная сумма существует, то она существует и для всего множества. Отметим также, что обобщенная сумма существует для любого конечного множества элементов (она равна обычной их сумме). Также обобщенная сумма любого количество нулей равна нулю, поэтому достаточно доказывать существование обобщенной суммы только для множеств ненулевых элементов.

Обобщенная сумма также существует для любого множества элементов такого, что в множестве их младших степеней нет наименьшего элемента. Их обобщенной суммой, является, например, ноль. Это вытекает из того, что в таком множестве $A$, если все элементы, кроме, быть может, конечного числа, лежат в некотором фильтрующем множестве $U_{g}$, то в нем лежат все элементы множества $A$. 
Пусть теперь $\left\{r_{\alpha} \mid \alpha \in \Omega\right\}-$ произвольный набор элементов кольца $R$. Докажем, что для него существует обобщенная сумма. Без ограничения общности можно считать, что все элементы $r_{\alpha}$ отличны от нуля. Если множество $\left\{r_{\alpha}\right\}$ можно разбить на два подмножества, одно из которых конечно, а для второго выполнено условие, что в множестве младших степеней нет наименьшего элемента, то существование обобщенной суммы уже доказано. Поэтому можно предполагать, что в множестве $\left\{r_{\alpha}\right\}$, если из него изъять любое конечное число элементов, всегда найдется элемент с наименьшей младшей степенью.

Тогда построим цепочку элементов: $r_{1}$ - элемент с наименьшей младшей степенью из всех $\left\{r_{\alpha}\right\}, r_{2}$ - элемент с наименьшей младшей степенью из оставшихся и так далее. Цепочка младших степеней этих элементов является монотонно неубывающей. Если эта цепочка стабилизируется на какой-то степени $g$, то в качестве обобщенной суммы всего множества $\left\{r_{\alpha}\right\}$ можно взять сумму всех тех элементов, младшая степень которых строго меньше $g$ (их конечное число).

Если же эта цепочка не стабилизируется, то можно объединить в этой цепочке элементы с одинаковыми младшими степенями (просуммировав их, поскольку их для каждой младшей степени конечное число) и получить цепочку со строго возрастающей последовательностью младших степеней, к которой можно применить условие. Оставшаяся часть утверждения проверяется непосредственно.

Докажем еще одно важное предложение, позволяющее строить дополнительные примеры обобщенных мальцевских колец.

Предложение 3. Если в условиях предложения 1 сильно фильтрованные кольцуа $S$ u $R$ являются ещче и обобщенными мальцевскими кольцами (в смысле фильтраций $U u V$ ), то и для построенной в предложении новой фильтрации $W$ кольцьо $S$ будет обобщенным мальиевским.

Доказательство. Воспользуемся леммой 5. Пусть $s_{1}, s_{2}, \ldots$ - последовательность ненулевых элементов кольца $S$ такая, что последовательность их младших степеней $f_{1}, f_{2}, \ldots$ (в смысле фильтрации $\left\{W_{f}\right\}$ ) строго возрастает. Пусть $g_{1}, g_{2}, \ldots$ - это последовательность их младших степеней в смысле фильтрации $\left\{U_{g}\right\}$. Эта последовательность также является неубывающей, но может возрастать нестрого. В случае, если эта последовательность не стабилизируется, легко видеть, что можно взять обобщенную сумму элементов $s_{1}, s_{2}, \ldots$ в смысле мальцевского кольца $S$ с фильтрацией $\left\{U_{g}\right\}$, она же будет и обобщенной суммой в смысле мальцевского кольца $S$ с фильтрацией $\left\{W_{f}\right\}$.

Если же последовательность $g_{1}, g_{2}, \ldots$ стабилизируется на элементе $g$, то можно, выкинув из обобщенной суммы конечное число членов с младшей степенью, меньшей $g$, и домножив все элементы цепочки с одной стороны на какой-нибудь сильно обратимый элемент с младшей степенью $g^{-1}$, считать, что все члены последовательности $s_{1}, s_{2}, \ldots$ имеют единичную младшую степень (в смысле фильтрации $U$ ). После этого достаточно перейти к факторкольцу $R$, рассмотреть соответствующую цепочку $r_{1}, r_{2}, \ldots$ (образ при каноническом гомоморфизме) и получить ее обобщенную сумму в смысле кольца $R$. Любой прообраз этой обобщенной суммы в кольце $S$ будет обобщенной суммой исходных элементов $s_{1}, s_{2}, \ldots$ (проверяется непосредственно).

Новые обобщенные мальцевские кольца можно строить также как факторкольца уже построенных.

Предложение 4. Пусть $R$ - обобщенное мальцевское кольцо с фильтрацией $\left\{U_{g}\right\}$ и кольцุом коэффициентов $A$. Пусть $I-$ двусторонний собственньй идеал кольца $R$ такой, 
что для любого набора элементов $\left\{r_{\alpha} \in I \mid \alpha \in \Omega\right\}$ этого идеала, существует по крайней мере одна их обобщенная сумма, лежащая в том же идеале I. Тогда факторкольио $R / I$ также является обобщенным мальцевским кольияом с фильтрацией $\left\{V_{g}\right\}$, где каждое фильтрующее множество $V_{g}$ является образом при каноническом гомоморфизме соответствующего множества $U_{g}$. Кольияо коэффициентов при этом будет изоморфно $A / \lambda(I)$.

Доказательство. Докажем, что $\left\{V_{g}\right\}-$ строго исчерпывающая сверху фильтрация. Пусть $s$ - произвольный ненулевой элемент кольца $R / I$. Достаточно доказать, что в множестве всех элементов $g$ группы $G$ таких, что $s \in V_{g}$, есть наибольший. Пусть $s=r+I$, где $r$ - какой-то элемент кольца $R$, не лежащий в $I$. Тогда достаточно доказать, что в множестве всех элементов $g$ группы $G$ таких, что $r \in U_{g}+I$, есть наибольший. Или, что то же самое, что среди элементов вида $r+j$, где $j$ лежит в идеале $I$, есть элемент с наибольшей младшей степенью. Применим лемму Цорна.

Действительно, пусть $r+j_{1}, r+j_{2}, r+j_{3}, \ldots-$ бесконечная последовательность элементов кольца $R$ со строго увеличивающимися младшими степенями $g_{1}, g_{2}, g_{3}, \ldots$, причем все элементы $j_{n}$ лежат в идеале $I$. Отметим, что для всякого натурального $n$ справедливо включение $j_{n+1}-j_{n}=\left(r+j_{n+1}\right)-\left(r+j_{n}\right) \in U_{g_{n}}$. По условию для элементов $\left(j_{2}-j_{1}\right),\left(j_{3}-j_{2}\right), \ldots$ должна найтись хотя бы одна обобщенная сумма $j$, лежащая в $I$. Непосредственно проверяется, что элемент $r+j_{1}+j$ имеет младшую степень, большую, чем все $g_{n}$ и, таким образом, является искомой верхней гранью последовательности $r+j_{1}, r+j_{2}, r+j_{3}, \ldots$, что и позволяет применить лемму Цорна.

Таким образом, доказано, что $\left\{V_{g}\right\}-$ строго исчерпывающая сверху фильтрация. Оставшиеся условия определения мальцевского кольца проверяются тривиально. Действительно, если $r$ - сильно обратимый элемент в кольце $R$ с младшей степенью $g$, то $r+I-$ сильно обратимый элемент в кольце $R / I$ с той же младшей степенью, поскольку выполнены включения $r+I \in V_{g}$ и $r^{-1}+I \in V_{g-1}$. Если $\left\{s_{\alpha}\right\}-$ набор элементов кольца $R / I$, а $\left\{r_{\alpha}\right\}$ - набор их прообразов в кольце $R$ таких, что младшая степень элемента $s_{\alpha}$ в кольце $R / I$ совпадает с младшей степенью элемента $r_{\alpha}$ в кольце $R$ для всех $\alpha$, то образ обобщенной суммы элементов $r_{\alpha}$ в кольце $R / I$ будет обобщенной суммой элементов $s_{\alpha}$ (проверяется непосредственно).

Замечание 3. Аналог этого утверждения для мальцевских колец не верен. Так, ниже будет приведен пример факторкольца кольца рядов Лорана, которое является обобщенным мальцевским кольцом, но не является мальцевским кольцом.

\section{6. Мальцевские кольца}

Пусть $R$ - мальцевское кольцо, а $A-$ его кольцо коэффициентов. Пусть $\pi-$ вложение кольца $A$ в кольцо $R$, как в определении мальцевского кольца. Тогда для каждого правого идеала $B$ кольца $A$ обозначим $\mu(B)$ правый идеал $\pi(B) R$. Из условия (iv) определения вытекает, что $\pi(B) R \cap U_{1}=\pi(B) U_{1}$, поэтому $\lambda(\mu(B))=B$. Отображение $\mu$ осуществляет вложение решетки правых идеалов кольца $A$ в решетку правых идеалов кольца $R$ (это вложение является гомоморфизмом относительно решеточных операций сложения и пересечения, в том числе и бесконечных сумм и пересечений). Легко видеть, что для любого главного правого идеала $a A$ кольца коэффициентов выполнено равенство $\mu(a A)=\pi(a) R$. 
Замечание 4. Отображение $\mu$, в отличие от отображения $\lambda$, определено не симметрично относительно умножения справа или слева. Можно было бы определить его для левых коэффициентов и тогда оно осуществляло бы вложение решетки левых идеалов. Кроме того, наличие такого отображения требует выполнения условия (iv) определения мальцевского кольца и само отображение зависит от выбора конкретного биективного отображения $\pi$ из $A$ в $R$.

Предложение 5. Пусть $R$-мальцевское кольцьо, a $A$ - его кольцุо коэффициентов, тогда кольц̧о $R$ является телом тогда и только тогда, когда кольичо А является телом.

Доказательство. В одну сторону утверждение доказано в предложении 2. Пусть теперь $R$ - тело. Тогда кольцо $A$ является областью, поскольку вкладывается в тело $R$. Пусть $a$ - произвольный ненулевой элемент кольца $A$. Элемент $\pi(a)$ обратим в теле $R$, пусть $r$ - его обратный. По лемме 1 выполнено равенство $\operatorname{deg} r=\operatorname{deg} \pi(a) \operatorname{deg} r=\operatorname{deg} 1_{R}=1$, откуда получаем, что $r \in U_{1}$, и тогда можно рассмотреть $\hat{r}$, свободный член элемента $r$. С учетом того, что произведение свободных членов равно свободному члену произведения, получаем, что $a \hat{r}=1$. Аналогично $\hat{r} a=1$, что и требовалось доказать.

Можно получить для мальцевского кольца критерии артиновости и нетеровости, которые также были известны ранее для колец косых рядов Лорана, колец псевдодифференциальных операторов и колец рядов Мальцева-Неймана.

Предложение 6. Пусть $R$ - мальцевское кольцуо, a $A$-его кольцуо коэффициентов, тогда кольијо $R$ нетерово справа тогда и только тогда, когда кольцо $A$ нетерово справа.

Доказательство. Если кольцо $R$ нетерово справа, то, поскольку решетка правых идеалов кольца $A$ инъективно вкладывается в решетку правых идеалов кольца $R$ (с помощью отображения $\mu$ ), и кольцо $A$ нетерово справа.

Пусть теперь $A-$ нетерово справа кольцо. Допустим, что кольцо $R$ не нетерово справа, тогда в нем существует бесконечная строго возрастающая цепочка правых идеалов $B_{1}, B_{2}, B_{3}, \ldots$ Рассмотрим возрастающую цепочку правых идеалов $\lambda\left(B_{1}\right), \lambda\left(B_{2}\right), \ldots$ в кольце $A$. По условию кольцо $A$ нетерово справа, поэтому существует такое натуральное число $k$, что $\lambda\left(B_{n}\right)=\lambda\left(B_{k}\right)$ для всех $n>k$. Но кольцо $A$ нетерово справа, поэтому все правые идеалы $\lambda\left(B_{n}\right)$ конечно порождены. Тогда идеал $\lambda\left(B_{k}\right)$ порождается конечным числом элементов кольца $A$, которые являются свободными членами каких-то элементов $\left\{c_{i}\right\}$ из множества $U_{1} \cap B_{k}$. Тогда для любого $n>k$ к правым идеалам $B_{n}$ и $B_{k}$ и к набору элементов $\left\{c_{i}\right\}$ применима лемма 4 , откуда вытекает, что все правые идеалы $B_{n}$ совпадают при $n>k$, что противоречит выбору цепочки $B_{1}, B_{2}, B_{3}, \ldots$ Полученное противоречие завершает доказательство.

Предложение 7. Пусть $R$ - мальцеевское кольц̧о, a $A$-его кольијо коэффициентов, тогда кольц̧о $R$ артиново справа тогда и только тогда, когда кольцо А артиново справа.

Доказательство. Доказательство полностью аналогично доказательству предложения 6.

Замечание 5. Можно отметить, что доказательство предложений 6 и 7 использует условие (iv) определения мальцевского кольца только для доказательства в одну сторону. Таким образом, если кольцо коэффициентов $A$ нетерово или артиново, то кольцо $R$ нетерово или артиново справа, даже если $R$ - обобщенное мальцевское кольцо.

Предложение 8. Если в условиях предложения 3 обобщенные мальцевские кольца $S$ u $R$ являются ещуе и мальцевскими кольцами (в смысле фильтраций $U$ u V), то и для построенной в предложении новой фильтрации $W$ кольц̧о $S$ будет мальцевским. 
Доказательство. Необходимое вложение $\pi$ кольца $A$ в кольцо $S$ строится путем простой композиции вложений $\pi_{1}$ из $A$ в $R$ и $\pi_{2}$ из $R$ в $S$, которые существуют по условию.

Надо доказать, что для любого $a$ из $A$ выполнены равенства $\pi(a) S \cap W_{1}=\pi(a) W_{1}$, $S \pi(a) \cap W_{1}=W_{1} \pi(a)$. Достаточно доказать первое, второе доказывается аналогично. Включение $\pi(a) W_{1} \subseteq \pi(a) S \cap W_{1}$ очевидно, докажем обратное. Пусть $s-$ произвольный элемент из множества $\pi(a) S \cap W_{1}$. Он лежит также в $U_{1}$ и его образ при каноническом гомоморфизме $U_{1}$ на $R$ лежит в $\pi_{1}(a) R \cap V_{1}$. По условию это означает, что этот образ лежит в $\pi_{1}(a) V_{1}$, то есть имеет вид $\pi_{1}(a) v_{1}$. Это означает, что выполнено равенство $s \in \pi(a) \pi_{2}\left(v_{1}\right)+U_{1}$. Пусть $g-$ младшая степень элемента $s-\pi(a) \pi_{2}\left(v_{1}\right)$ (в смысле фильтрации $U)$, а $s_{g}$ - произвольный сильно обратимый элемент с младшей степенью $g$. Отметим, что $g$ строго больше единицы. Тогда элемент $s^{\prime} \equiv\left(s-\pi(a) \pi_{2}\left(v_{1}\right)\right) s_{g}^{-1}$ лежит в $U_{1}$. С другой стороны, этот же элемент лежит и в $\pi(a) S$, поэтому, по условию, он лежит и в $\pi(a) U_{1}$. Но тогда элемент $s^{\prime} s_{g}$ лежит в $\pi(a) U_{1} \subseteq \pi(a) W_{1}$, и поэтому элемент $s=\pi(a) \pi_{2}\left(v_{1}\right)+s^{\prime} s_{g}$ также лежит в $\pi(a) W_{1}$, что и требовалось доказать.

\section{7. Примеры мальцевских колец}

Назовем упорядоченное множество вполне упорядоченным снизу, если в любом его непустом подмножестве найдется наименьший элемент.

Одним из основных примеров мальцевского кольца является кольцо рядов МальцеваНеймана (а также кольца рядов Лорана и кольца косых рядов Лорана как его частные случаи).

Пусть $A-$ кольцо и $G$ - упорядоченная группа, и пусть имеется отображение $\varphi: G \rightarrow \operatorname{Aut}(A)$. Для любого $g \in G$ положим $\varphi_{g}=\varphi(g)$. Кроме того, пусть имеется некоторое отображение $\varepsilon: G \times G \rightarrow U(A)$, сопоставляющее каждой паре элементов группы $G$ какой-либо обратимый элемент кольца $A$. Обозначим через $A((G, \varphi, \varepsilon))$ множество всех формальных сумм $r=\sum_{g \in G} r_{g} \bar{g}, r_{g} \in A$, таких что множества

$$
\operatorname{Supp}(r)=\left\{g \in G \mid r_{g} \neq 0\right\}
$$

вполне упорядочены снизу. На множестве $A((G, \varphi, \varepsilon))$ можно ввести структуру кольца, определив сложение обычным образом и задав умножение при помощи правила перемножения одночленов

$$
(a \bar{g}) \times(b \bar{h})=\left(a \varphi_{g}(b) \varepsilon(g, h)\right) \overline{g h} .
$$

Умножение может быть распространено на бесконечные формальные суммы одночленов, поскольку в сумме, задающей коэффициент при данном $\bar{g}$, лишь конечное число членов будет отлично от нуля. Вообще говоря, необходимо наложить некоторые условия на отображения $\varphi$ и $\varepsilon$, чтобы это умножение было ассоциативно и чтобы в кольце существовала единица. Для любых $g, h, f$ из группы $G$ и любого $a$ из кольца $A$ должны быть выполнены равенства

(i) $\varepsilon(g, h) \varepsilon(g h, f)=\varphi_{g}(\varepsilon(h, f)) \varepsilon(g, h f)$,

(ii) $\varphi_{g h}(a)=(\varepsilon(g, h))^{-1} \varphi_{g}\left(\varphi_{h}(a)\right) \varepsilon(g, h)$.

Второе условие может быть переписано в виде

$$
\varphi_{g h}=\delta(g, h) \varphi_{g} \varphi_{h},
$$

где $\delta(g, h)$ - внутренний автоморфизм кольца $A$, индуцированный элементом $\varepsilon(g, h)$. 
Непосредственно проверяется следующее утверждение.

Предложение 9. Множество $R=A((G, \varphi, \varepsilon))$, где $\varphi$ и $\varepsilon$ заданы, как в определении кольца рядов Мальцева-Неймана, является кольиом, причем единицей в нем является одночлен $1_{R}=(\varepsilon(1,1))^{-1} \overline{1_{G}}$, где $1_{G}-$ единица группь $G$.

Если положить $G$ равной свободной циклической группе $\langle x\rangle$, положить $e(g, h) \equiv 1$ и $\varphi_{x^{n}}=\varphi^{n}$, где $\varphi-$ фиксированный автоморфизм кольца коэффициентов $A$, то условия определения будут выполнены автоматически и получится кольцо $A((x, \varphi))$ косых рядов Лорана, являющееся, таким образом, частным случаем кольца рядов Мальцева-Неймана.

Определенное выше понятие мальцевского кольца является дальнейшим обобщением конструкции колец рядов Мальцева-Неймана и включает в себя также и кольца псевдодифференциальных операторов, свойства которых близки к свойствам колец рядов Мальцева-Неймана (и, в частности, колец косых рядов Лорана).

Предложение 10. Пусть $R=A((G, \varphi, \varepsilon))$ - кольичо рядов Мальцева-Неймана. Тогда оно является мальцевским кольиом, причем его кольцьо коэффициентов (как мальцевского кольйа) изоморфно кольиу А.

Доказательство. Для каждого элемента $g$ из $G$ обозначим $U_{g}$ множество таких формальных сумм $r$ из $R$, что все элементы $\operatorname{Supp}(R)$ больше либо равны $g$.

Условие (i) определения мальцевского кольца проверяется непосредственно, для проверки условия (ii) достаточно заметить, что для любого $g$ из $G$ элемент $1_{A} \bar{g}$ обратим и обратный к нему равен $\left(\varepsilon\left(g^{-1}, g\right)\right)^{-1} \overline{g^{-1}}$. Легко видеть, что младшая степень элемента $r$ всегда совпадает с наименьшим элементом множества $\operatorname{Supp}(r) \subset G$.

Проверим условие (iii). Пусть $\left\{r_{\alpha} \mid \alpha \in \Omega\right\}$ - произвольное множество элементов кольца $R$. Будем строить формальную сумму $s$, являющуюся обобщенной суммой элементов $\left\{r_{\alpha}\right\}$. Для всех $g$ из $G$ таких, что не более чем конечное число элементов $\left\{r_{\alpha}\right\}$ имеет степень меньше или равную $g$, положим $s_{g}$ равным сумме коэффициентов $\left\{r_{\alpha}\right\}$ при $\bar{g}$ (эта сумма определена корректно, поскольку лишь конечное число этих коэффициентов отлично от нуля). Для всех остальных $g$ из $G$ положим $s_{g}=0$. Тогда формальная сумма $s=\sum s_{g} \bar{g}$ и будет искомой обобщенной суммой $\left\{r_{\alpha}\right\}$. Докажем это.

Вначале докажем, что формальная сумма $s=\sum s_{g} \bar{g}$ лежит в кольце $R$, то есть, что $\operatorname{Supp}(s)$ - вполне упорядоченное снизу множество. Действительно, предположим, что это не так. Тогда в нем существует непустое подмножество без наименьшего элемента, и следовательно, можно построить бесконечную строго убывающую цепь элементов $g_{1}>g_{2}>g_{3}>\ldots$, лежащих в $\operatorname{Supp}(s)$. По построению $s$ существует лишь конечное число элементов $r_{\alpha}$ с младшей степенью, меньшей либо равной $g_{1}$. Пусть это будет набор $r_{1}, r_{2}, \ldots, r_{n}$. Поскольку коэффициент $s_{g_{i}}$ отличен от нуля для всех $i$ и коэффициент $s_{g_{i}}$ равен сумме коэффициентов при $\bar{g}_{i}$ всех $r_{j}, 1 \leqslant j \leqslant n$, для каждого натурального $i$ найдется хотя бы одно $j$ такое, что коэффициент $r_{j}$ при $\bar{g}_{i}$ отличен от нуля. Поскольку $i$ пробегает весь натуральный ряд, а $j$ может принимать лишь конечное число значений, одно из значений $j$ будет встречаться бесконечное число раз, без ограничения общности можно считать, что это $j=1$. Тогда в последовательности $g_{1}, g_{2}, \ldots$ можно выделить подпоследовательность $g_{i_{1}}, g_{i_{2}}, \ldots$, для которой коэффициент $r_{1}$ при $g_{i_{k}}$ отличен от нуля для всех $k$. Но тогда $\operatorname{Supp}\left(r_{1}\right)$ не является вполне упорядоченным снизу множеством, что противоречит включению $r \in R$. 
Остается доказать, что элемент $s$ удовлетворяет определению обобщенной суммы. Действительно, пусть для некоторого $g \in G$ все элементы $\left\{r_{\alpha}\right\}$ (кроме конечного числа $\left.r_{1}, \ldots, r_{n}\right)$ лежат в $U_{g}$. Тогда разность

$$
t=\left(s-\sum_{i=1}^{n} r_{n}\right)
$$

должна лежать в $U_{g}$. Действительно, допустим, что это не так, тогда младшая степень $t$ должна быть меньше $g$. Пусть младшая степень $t$ равна $h$. Коэффициент $s$ при $\bar{h}$ по построению равен сумме таких же коэффициентов $\sum_{i=1}^{n} r_{n}$, поэтому коэффициент $t$ при $\bar{h}$ равен нулю, что противоречит выбору $h$. Полученное противоречие завершает проверку условия (iii).

Остается проверить условие (iv). Докажем вначале существование инъективного кольцевого гомоморфизма $\pi$ из $A$ в $R$. Обозначим $\varepsilon(1,1)$ через $e$. Положим $\pi(a)=a e^{-1} \overline{1_{G}}$, при этом отображении единица $1_{A}$ кольца $A$ переходит в единицу $1_{R}=e^{-1} \overline{1_{G}}$ кольца $R$. Очевидным образом, $\pi(a+b)=\pi(a)+\pi(b)$, проверим, что $\pi(a b)=\pi(a) \times \pi(b)$. Действительно,

$$
\pi(a) \times \pi(b)=\left(a e^{-1} \overline{1_{G}}\right) \times\left(b e^{-1} \overline{1_{G}}\right)=a e^{-1} \varphi_{1}\left(b e^{-1}\right) e \overline{1_{G}} .
$$

С другой стороны,

$$
b e^{-1} \overline{1_{G}}=1_{R} \times\left(b e^{-1} \overline{1_{G}}\right)=e^{-1} \varphi_{1}\left(b e^{-1}\right) e \overline{1_{G}},
$$

так что

$$
b e^{-1}=e^{-1} \varphi_{1}\left(b e^{-1}\right) e .
$$

Поэтому

$$
\pi(a) \times \pi(b)=a e^{-1} \varphi_{1}\left(b e^{-1}\right) e \overline{1_{G}}=a b e^{-1} \overline{1_{G}}=\pi(a b),
$$

что и требовалось доказать.

По построению $\pi$ видно, что $\pi(A) \cap U_{1+}=0$. Докажем, что $\pi(A)+U_{1+}=U_{1}$. Действительно, каждый элемент $r$ из $U_{1}$ может быть представлен в виде суммы $a \overline{1_{G}}+s$, где $a$ - элемент кольца $A$, а $s$ лежит в $U_{1+}$. Но $a \overline{1_{G}}=\pi(a e)$, что и доказывает требуемое равенство $\pi(A)+U_{1+}=U_{1}$. Тогда получаем, что кольцо $A$ изоморфно $U_{1} / U_{1+}$ и существует требуемое вложение $\pi$.

Остается проверить, что $\pi(a) R \cap U_{1}=\pi(a) U_{1}$ и $R \pi(a) \cap U_{1}=U_{1} \pi(a)$. Докажем только первое соотношение, второе доказывается совершенно аналогично. Пусть $s=\pi(a) r \in \pi(a) R \cap U_{1}$. Тогда если $r$ - это формальная сумма одночленов $r_{g} \bar{g}$, то $s-$ формальная сумма одночленов $a e^{-1} \varphi_{1}\left(r_{g}\right) \varepsilon(1, g) \bar{g}$. Из того, что $s$ лежит в $U_{1}$, следует, что все одночлены $\pi(a) \times r_{g} \bar{g}=a e^{-1} \varphi_{1}\left(r_{g}\right) \varepsilon(1, g) \bar{g}$ равны нулю при $g<1$. Но это означает, что $0=\pi(a)\left(r-r^{\prime}\right)$, где $r^{\prime}-$ формальная сумма тех одночленов $r_{g} \bar{g}$, у которых $g \geqslant 1$. При этом $r^{\prime}$ лежит в $U_{1}$, поэтому $s=\pi(a) r^{\prime} \in \pi(a) U_{1}$, что и требовалось доказать.

Помимо колец рядов Мальцева-Неймана, под определение мальцевского кольца попадают также кольца косых рядов Лорана с косым дифференцированием, которые включают в себя кольца косых рядов Лорана и кольца псевдодифференциальных операторов. Формально строгое построение кольца косых рядов Лорана с косым дифференцированием слишком длинно, поэтому здесь будет приведена только формулировка без доказательства. 
Пусть $A-$ кольцо, $\varphi-$ его автоморфизм, а $\delta-\varphi^{-1}$-дифференцирование, то есть эндоморфизм абелевой группы по сложению $A^{+}$, удовлетворяющий условию

$$
\delta(a b)=\delta(a) b+\varphi^{-1}(a) \delta(b)
$$

для всех $a$ и $b$ из $A$. Тогда существует и единственно кольцо $A((x, \varphi, \delta))$, совпадающее как абелева группа по сложению с кольцом рядов Лорана $A((x))$, в котором умножение удовлетворяет соотношениям

$$
x^{-1} a=\varphi^{-1}(a) x^{-1}+\delta(a), \quad\left(a x^{n}\right)\left(1 x^{m}\right)=a x^{n+m}, \quad\left(a x^{0}\right)\left(b x^{n}\right)=(a b) x^{n},
$$

и кроме того, младшая степень произведения двух рядов всегда больше или равна сумме их младших степеней. Под младшей степенью ряда здесь подразумевается степень младшего члена ряда.

Непосредственно проверяется, что кольцо косых рядов Лорана с косым дифференцированием также является мальцевским кольцом, если в качестве группы $G$ взять свободную циклическую группу $\langle x\rangle$, а в качестве $U_{x^{n}}$ взять множество всех рядов, младшая степень которых не ниже $n$. При этом его кольцо коэффициентов как мальцевское кольцо будет изоморфно $A$.

Конструкция кольца рядов Лорана допускает итерирование - можно рассмотреть кольцо итерированных рядов Лорана от $n$ переменных $A\left(\left(x_{1}\right)\right)\left(\left(x_{2}\right)\right) \ldots\left(\left(x_{n}\right)\right)$, определив его по индукции как кольцо обычных рядов Лорана от переменной $x_{n}$ с кольцом коэффициентов $A\left(\left(x_{1}\right)\right)\left(\left(x_{2}\right)\right) \ldots\left(\left(x_{n-1}\right)\right)$. При таком определении переменные неравноправны и их порядок существен. Свойства кольца итерированных рядов Лорана близки к свойствам кольца обычных рядов Лорана; можно показать, что на кольце итерированных рядов Лорана $A\left(\left(x_{1}\right)\right)\left(\left(x_{2}\right)\right) \ldots\left(\left(x_{n}\right)\right)$ можно ввести структуру мальцевского кольца так, чтобы его кольцо коэффициентов было изоморфно $A$. Конструкцию мальцевского кольца можно итерировать (при соблюдении некоторых условий), как показано в предложении 8.

Обобщенные мальцевские кольца можно получать также факторизацией других мальцевских (и обобщенных мальцевских) колец. Например, кольцо целых $p$-адических чисел может быть получено как факторкольцо кольца формальных степенных рядов $\mathbf{Z}[[x]]$ по идеалу, порожденному рядом $(x-p)$, а кольцо дробных $p$-адических чисел может быть получено как факторкольцо кольца рядов Лорана $\mathbf{Z}((x))$ по идеалу, порожденному рядом $(x-p)$. В силу предложения 4 кольцо дробных $p$-адических чисел является обобщенным мальцевским кольцом с кольцом коэффициентов $\mathbf{Z} / p \mathbf{Z}$. Аналогично можно рассмотреть и кольцо дробных $n$-адических чисел для любого целого $n>2$, которое будет обобщенным мальцевским кольцом с кольцом коэффициентов $\mathbf{Z} / n \mathbf{Z}$. Интересно отметить следующий факт.

Предложение 11. Пусть $A$ - кольичо, а $A((x))$ - кольцุо рядов Лорана над ним. Пусть $а$ какой-то центральный необратимый элемент кольца коэффищиентов $A$, не являющийся делителем нуля, а I - двусторонний идеал кольца $A((x))$, порожденный рядом $x-a$. Пусть $n$ - произвольное натуральное число. Тогда факторкольияо $A((x)) / I$ изоморфно факторкольиу $A((y)) / J$, где $J$ - двусторонний идеал кольца рядов Лорана $A((y))$, порожденный рядом $y-a^{n}$. 
Доказательство. Рассмотрим кольцевой гомоморфизм $\varphi$, который отображает кольцо $A((y))$ в кольцо $A((x))$, переводя $y$ в $x^{n}$, а бесконечные формальные суммы степеней $y$ в соответствующие бесконечные формальные суммы степеней $x^{n}$. Очевидно, $\varphi-$ кольцевое вложение. Очевидно также, что идеал $J$ при этом вложении переходит внутрь идеала $I$ (поскольку его порождающий элемент $y-a^{n}$ переходит в элемент $x^{n}-a^{n}$, лежащий в идеале $I)$. Докажем теперь, что идеал $J$ совпадает с прообразом идеала $I$ при гомоморфизме $\varphi$. Для этого достаточно доказать, что прообраз $\varphi^{-1}(I)$ идеала $I$ порождается центральным элементом $y-a^{n}$. В силу леммы 4 для этого достаточно доказать, что множество всех свободных членов всех рядов без отрицательных степеней из прообраза $\varphi^{-1}(I)$ лежит в идеале $a^{n} A$ кольца $A$. При вложении $\varphi$ свободные члены рядов сохраняются (а ряды без отрицательных степеней переходят в ряды без отрицательных степеней), поэтому достаточно доказать, что все свободные члены всех рядов без отрицательных степеней из пересечения $I \cap \varphi(A((y)))$ лежат в $a^{n} A$.

Обозначим образ $\varphi(A((y)))$ через $A\left(\left(x^{n}\right)\right)$ (поскольку он состоит из тех и только тех рядов в $A((x))$, у которых отличны от нуля только коэффициенты при степенях $x$, кратных $n)$. Пусть $f$ - ряд без отрицательных степеней, лежащий в $I \cap A\left(\left(x^{n}\right)\right)$, а $f_{0}-$ его свободный член. Тогда ряд $f$ имеет вид $f_{0}+f_{n} x^{n}+\ldots$ Предположим, что $f_{0}$ не лежит в $a^{n} A$. Пусть тогда $f_{0}=a^{k} b$, где $k$ - некоторое натуральное число, а элемент $b$ кольца $A$ не лежит в $a A$. По предположению, $k<n$. Тогда ряд $f-b\left(a^{k}-x^{k}\right)$ также лежит в $I$, причем его младший член равен $b x^{k}$, а младший коэффициент равен $b$. Однако, поскольку элемент $a$ не является делителем нуля, младший коэффициент любого ряда из идеала $I=(x-a) A((x))$ должен лежать в идеале $a A$. Полученное противоречие доказывает, что идеал $J$ совпадает с прообразом идеала $I$ при гомоморфизме $\varphi$.

Докажем теперь равенство

$$
A((x))=A\left(\left(x^{n}\right)\right)+I
$$

Действительно, всякий ряд $f=f_{m} x^{m}+f_{m+1} x^{m+1}+\ldots$ из $A((x))$ представляется в виде конечной суммы рядов $f^{(k)}=f_{m+k} x^{m+k}+f_{n+m+k} x^{n+m+k}+f_{2 n+m+k} x^{2 n+m+1}+\ldots$, где $k$ пробегает все значения от 0 до $n-1$. При этом ряд $f^{(k)}$ имеет вид $x^{k} g^{(k)}$, где $g^{(k)}-$ ряд из $A\left(\left(x^{n}\right)\right)$. Тогда ряд $f$ представляется в виде $f=f_{0}+x g^{(1)}+x^{2} g^{(2)}+\ldots+x^{n-1} g^{(n-1)} \in$ $f_{0}+a g^{(1)}+a^{2} g^{(2)}+\ldots+a^{n-1} g^{(n-1)}+I$, что и требовалось доказать.

Построим теперь изоморфизм $\bar{\varphi}$ между кольцами $A((y)) / J$ и $A((x)) / I$. Если $f-$ ряд из $A((y))$, то поставим элементу $f+J$ в соответствие элемент $\varphi(f)+I$. Из того, что кольцевой гомоморфизм $\varphi$ переводит идеал $J$ внутрь идеала $I$, следует, что соответствие $\bar{\varphi}$ определено корректно и не зависит от выбора конкретного ряда $f$. Из того, что идеал $J$ совпадает с прообразом идеала $I$, вытекает, что соответствие $\bar{\varphi}$ взаимно однозначно. Из того, что

$$
A((x))=A\left(\left(x^{n}\right)\right)+I=\varphi(A((y)))+I,
$$

вытекает, что образом кольцевого гомоморфизма $\bar{\varphi}$ является все кольцо $A((x)) / I$, что и требовалось доказать.

Из доказанного утверждения вытекает, в частности, что поле дробных $p$-адических чисел для всех натуральных $k$ изоморфно кольцу дробных $p^{k}$-адических чисел, при этом они неизоморфны как мальцевские кольца (например, потому, что у одного из них кольцо коэффициентов - поле $\mathbf{Z} / p \mathbf{Z}$, а у другого даже не область $-\mathbf{Z} / p^{k} \mathbf{Z}$ ). 


\section{Список литературы}

1. Benhissi A., Les anneaux de séries formelles. In: Queen's Papers in Pure and Applied Mathematics, 124. Queen's Univ., Kingston, 2003.

2. Bergman G. M., Conjugates and $n$th roots in Hahn-Laurents group rings. Bull. Malaysian Math. Soc. (1978) 1, №2, 29-41.

3. Brookfield G., Noetherian generalized power series rings. Commun. Algebra (2004) 32, №3, 919926.

4. Faith C., Algebra: rings, modules, and categories, I. Springer, Berlin, 1973.

5. Goodearl K. R., Centralizers in differential, pseudo-differential, and fractional differential operator rings. Rocky Mountain J. Math. (1983) 13, №4, 573-618.

6. Goodearl K. R., Small L. W., Krull versus global dimension in Noetherian PI-rings. Proc. Amer. Math. Soc. (1984) 92, №2, 175-178.

7. Goodearl K. R., Warfield R. B., An introduction to noncommutative Noetherian rings. Cambridge Univ. Press, Cambridge, 1989.

8. Liu Z., The ascending chain condition for principal ideals of rings of generalized power series. Commun. Algebra (2004) 32, №9, 3305-3314.

9. Liu Z., Ahsan J., The Tor-groups of modules of generalized power series. Algebra Colloq. (2005) 12, №3, 477-484.

10. Lorenz M., Division algebras generated by finitely generated nilpotent groups. J. Algebra (1983) 85, 368-381.

11. Makar-Limanov L., The skew field of fractions of the first Weyl algebra contains a free noncommutative subalgebra. Commun. Algebra (1983) 11, №17, 2003-2006.

12. Мальцев А. И., О вложении групповых алгебр в тела. Докл. АН СССР (1948) 60, 1499-1501.

13. Musson I., Stafford K., Malcev-Neumann group rings. Commun. Algebra (1993) 21, №6, 20652075.

14. Neumann B. H., On ordered division rings. Trans. Amer. Math. Soc. (1949) 66, 202-252.

15. Паршин А. Н., О кольце формальных псевдодифференциальных операторов. Труды Матем. ин-та им. В. А. Стеклова (1999) 224, 291-305.

16. Pommersheim J., Shahriari Sh., Unique factorization in generalized power series rings. Proc. Amer. Math. Soc. (2006) 134, №5, 1277-1287.

17. Ribenboim P., Semisimple rings and von Neumann regular rings of generalized power series. $J$. Algebra (1997) 198, №2, 327-338.

18. Risman L., Twisted rational functions and series. J. Pure Appl. Algebra (1978) 12, 181-199.

19. Schur I., Über vertauschbare lineare Differentialausdrücke. Sitzungsber. Berliner Math. Ges. (1905) $4,2-8$.

20. Smits T. H., Skew-Laurent series over semisimple rings. Delft. Progr. Rep. (1977) 2, 131-136.

21. Сонин К. И., Регулярные кольца рядов Лорана. Фундаментальная и прикладная математика (1995) 1, №1, 315-317.

22. Сонин К. И., Регулярные кольца косых рядов Лорана. Фундаментальная и прикладная математика (1995) 1, №2, 565-568.

23. Сонин К. И., Бирегулярные кольца рядов Лорана. Вестник Московского Университета, сер. матем. мех. (1997) №4, 22-24.

24. Sonin C., Krull dimension of Malcev-Neumann rings. Commun. Algebra (1998) 26, №9, 2915-2931.

25. Туганбаев А. А., Радикал Джекобсона кольца рядов Лорана. Фундаментальная и прикладная математика (2006) 12, №2, 209-216. 
26. Туганбаев Д. А., Цепные кольца рядов Лорана. Фундаментальная и прикладная математика (1997) 3, №3, 947-951.

27. Туганбаев Д. А., Цепные кольца косых рядов Лорана. Вестник Московского Университета, сер. матем. мех. (2000) №1, 51-55.

28. Туганбаев Д. А., Кольца косых рядов Лорана и кольца главных идеалов. Вестник Московского Университета, сер. матем. мех. (2000) №5, 55-57.

29. Tuganbaev D. A., Some ring and module properties of skew power series. In: Formal Power Series and Algebraic Combinatorics. Springer, Berlin, 2000, pp. 613-622.

30. Туганбаев Д. А., Полулокальные дистрибутивные кольца косых рядов Лорана. Фундаментальная и прикладная математика (2000) 6, №3, 913-921.

31. Туганбаев Д. А., Кольца псевдодифференциальных операторов и условия на цепи. Вестник Московского Университета, сер. матем. мех. (2002) №4, 26-32.

32. Tuganbaev D. A., Laurent series rings and pseudo-differential operator rings. J. Math. Sci. (2005) 128, №3, 2843-2893.

33. Туганбаев Д. А., Лорановские кольца. Фундаментальная и прикладная математика (2006) 12 , №3, 151-224.

Статья поступила 6.10.2006. 